\title{
Characteristics of patients with Alzheimer's disease who switch to rivastigmine transdermal patches in routine clinical practice
}

This article was published in the following Dove Press journal:

Patient Preference and Adherence

9 January 2013

Number of times this article has been viewed

\author{
Secundino López-Pousa' \\ Francisco Javier Arranz ${ }^{2}$ \\ 'Unit for Assessment of Memory \\ and Dementia, Institut d'Assistència \\ Sanitària de Girona, Salt, Girona, ${ }^{2} \mathrm{CNS}$ \\ Area, Medical Department and Health \\ Innovation, Esteve, Barcelona, Spain
}

Correspondence: Francisco Javier Arranz CNS Area, Medical Department and Health Innovation, Esteve, Avda Mare de Déu de Montserrat 22I, E-0804I

Barcelona, Spain

Tel +34934466000

Fax +34934466251

Email fjarranz@esteve.es
Background: The aim of this study was to assess the sociodemographic and clinical characteristics of patients with Alzheimer's disease who switched from any oral cholinesterase inhibitor to rivastigmine patches.

Methods: An observational, retrospective, multicenter study was conducted in patients with a diagnosis of Alzheimer's disease who had switched to rivastigmine patches within the previous year in the routine clinical practice of 150 neurologists. Sociodemographic, clinical, and therapeutic data were collected in one office visit. Stepwise logistic regression models were used to find associations.

Results: Data were obtained from a total of 1022 patients and their caregivers, and showed a mean age of $78.4 \pm 6.62$ years, $62.61 \%$ being women, and mostly having a family caregiver. The switch to rivastigmine patches was mainly instigated on the initiative of the physician $(82.39 \%)$ or on request of the caregiver $(21.23 \%)$ or patient $(10.37 \%)$. Reasons for the switch included improving ease of administration (56.65\%), tolerability (36.79\%), efficacy (31.60\%), and adherence $(18.59 \%)$. Prior treatment with oral rivastigmine versus donepezil or galantamine increased the probability of switching in order to improve ease of administration (odds ratio, oral rivastigmine versus donepezil 4.20, $P<0.0001$; odds ratio, oral rivastigmine versus galantamine $3.55, P<0.0001)$. Conversely, previous treatment with donepezil or galantamine produced an approximate four-fold increase in the odds of switching due to lack of efficacy. A higher level of education as well as more concomitant diseases increased the probability of switching because of intolerance.

Conclusion: Improved ease of administration was the main reason for switching to transdermal rivastigmine. Other reasons involved in the decision to switch to rivastigmine patches included sociodemographic and clinical characteristics, including the educational level of patients and caregivers, number of concomitant diseases, and previous treatment for Alzheimer's disease.

Keywords: Alzheimer's disease, cholinesterase inhibitors, donepezil, galantamine, rivastigmine transdermal patches, adherence

\section{Introduction}

To date, there are no causal or disease-modifying therapeutic options for Alzheimer's disease (AD), and until now, only the cholinesterase inhibitors galantamine, rivastigmine, and donepezil, as well as the N-methyl D-aspartate receptor antagonist, memantine, have been approved for the symptomatic treatment of the dementia. ${ }^{1}$ These drugs may initially improve cognition and slow down the clinical progression of AD dementia, but they are not capable of blocking the underlying pathological processes of the disease, including amyloid accumulation, tau protein aggregation, synaptic loss, and neuronal death. ${ }^{1}$ 
The cholinesterase inhibitor, rivastigmine, is approved for the symptomatic treatment of mild to moderate AD and Parkinson's disease dementia, ${ }^{2}$ and can be administered by transdermal patches or orally as capsules or solution. ${ }^{3}$ Rivastigmine shows a dose-dependent efficacy pattern across its therapeutic oral dose range of 6-12 mg/day. Nevertheless, most patients on rivastigmine are not able to tolerate doses in excess of $6 \mathrm{mg} / \mathrm{day}^{4}$

Transdermal rivastigmine patches provide steadier continuous drug delivery, with the potential to offer an efficacious level of drug exposure while avoiding the peaks and troughs associated with side effects (mainly nausea and vomiting). ${ }^{2,3,5,6}$ This may be particularly important in AD patients with low body weight, who may be more susceptible to these adverse events. ${ }^{3}$ It has been demonstrated that the $9.5 \mathrm{mg} / 24$-hour rivastigmine patch provides efficacy similar to that of the highest-dose rivastigmine capsule (12 mg/day) but with a superior tolerability profile. ${ }^{2}$ Therefore, in the case of rivastigmine, the transdermal route of delivery provides considerable advantages, because it maintains the same efficacy as the oral route with a three-fold lower incidence of adverse effects, thereby allowing more patients to attain optimal therapeutic doses. Moreover, rivastigmine patches can be administered once daily at any time of the day and independently of food, while conventional capsules have to be administered twice daily with meals. ${ }^{5}$ Importantly, the results of recent studies have suggested that treatment with the transdermal patch may improve adherence, potentially leading to an increase in treatment benefits for patients with dementia of the Alzheimer's type. .,7, $^{-1}$

Transdermal delivery also brings some additional advantages inherent in this route of administration, related to better comfort for the caregiver and avoidance of accidental overdose. ${ }^{9}$ Thus, in the 24-week, randomized, double-blind, double-dummy, placebo and active-controlled IDEAL (Investigation of TransDermal Exelon in ALzheimer's disease) trial to investigate once-daily rivastigmine patches versus twice-daily capsules in patients with moderate AD, more than $70 \%$ of caregivers preferred the patch to the capsules overall. The patch was preferred to capsules with respect to ease of use $(P<0.0001)$ and ease of adherence to the administration schedule $(P<0.0001)$. Caregivers indicated greater satisfaction overall $(P<0.0001)$ and less interference with daily life $(P<0.01)$ with the patch versus capsules. ${ }^{9}$

However, to date, only studies comparing rivastigmine patches versus placebo or rivastigmine capsules have been carried out, ${ }^{9}$ while the advantages versus the oral solution and, importantly, versus other drugs that are also administered as a single-daily dose, such as donepezil or galantamine, have not been evaluated. The advantages of the rivastigmine patches over the capsules may not apply to these other treatments, so the reasons for switching from rivastigmine capsules to rivastigmine patches may be different from those that bring about the switch from rivastigmine oral solution or from other cholinesterase inhibitors. For instance, it was shown that caregivers of $\mathrm{AD}$ patients treated with donepezil were more satisfied with the easier to use orally disintegrating tablet formulation than with the film-coated tablets. ${ }^{10}$

In this context, the objective of this observational, retrospective, multicenter study was to ascertain the clinical and sociodemographic characteristics of patients with $\mathrm{AD}$ who switched from oral rivastigmine or other cholinesterase inhibitors to rivastigmine patches, as well as the practical reasons leading to the switch. As a secondary objective, we wanted to determine the pattern of switch to rivastigmine patches in routine clinical practice, given that a standard procedure does not exist to date, although it has been suggested that a washout period should be allowed when the switch is carried out because of intolerance to a previous cholinesterase inhibitor.

\section{Materials and methods}

Between April and September 2010, an observational, retrospective, multicenter study was conducted in Spain during outpatient office visits in the routine clinical practice of 150 Spanish neurologists. Prior to participation, patients provided written informed consent if they were determined by the investigator to be mentally competent. If the patient was not able to provide written informed consent, this was obtained from an authorized representative on the patient's behalf. The study protocol was approved by the Ethics Committee of Human Experimentation in Girona, Spain, and all study procedures were in accordance with the ethical standards laid down in the Declaration of Helsinki as revised in 2000.

Each investigator consecutively enrolled 10 patients fulfilling the following inclusion criteria: diagnosis of AD according to DSM-IV-TR (Diagnostic and Statistical Manual of Mental Disorders, Fourth Edition, Text revision) criteria, with Mini-Mental State Examination (MMSE) scores between 24 and 10 and had been switched to transdermal rivastigmine patches (within the previous year) from any other oral cholinesterase inhibitor (including rivastigmine capsules or solution), whether in combination with memantine or not. Patients with other types of dementia or those treated with transdermal rivastigmine patches as the first option were excluded from the study. 
Sociodemographic (including caregiver data), clinical (AD and concomitant diseases) and therapeutic (other treatments, programs, reasons, instigator of switch) data were obtained in the context of one office visit.

\section{Statistical analysis}

Frequency tables and percentages were obtained for categorical variables, while measures of central tendency and dispersion were calculated for continuous variables (mean, standard deviation, standard error, minimum and maximum, and $95 \%$ confidence intervals, median and quartiles Q1 and Q3).

The Chi-square test was used to compare qualitative variables between groups (severity of AD depending on family history of the disease), while analysis of variance was used to compare quantitative variables (severity of $\mathrm{AD}$ depending on years of evolution). In order to find associations between reasons for the switch and other variables, a stepwise logistic regression model was applied. Finally, the Hosmer-Lemeshow test was performed to establish the goodness of fit of the models. In all cases, statistical significance was defined as $P$ values less than 0.05 , and the statistical software package $\mathrm{SAS}^{\circledR}$ version 9.2 (Cary, NC) was used.

\section{Results}

In total, 1408 patients were recruited, of whom 1022 $(72.59 \%)$ fulfilled the selection criteria and were included in the study, thus being considered the evaluable sample. The sample included more women (62.61\%) than men (37.39\%), with a mean age of 78.4 years and a mean weight of $68.4 \mathrm{~kg}$ (Table 1). Most were not institutionalized (82.83\%), living accompanied (90.58\%) and having a caregiver (99.02\%), usually their children $(43.32 \%)$ or life partner $(40.37 \%)$. In total, $14.92 \%$ of the patients had been hospitalized within the previous year, with a median of one admission and 10 days of hospitalization.

Regarding clinical characteristics, the mean disease evolution time was 2.46 years, with $27.17 \%$ of patients having a family history of AD. The severity of their AD (median MMSE score 20) was in most cases mild (39.14\%) or moderate $(44.72 \%)$, and only $16.14 \%$ of patients had moderately severe $\mathrm{AD}$. The most frequent concomitant diseases were vascular (36.88\%), musculoskeletal (33.78\%), endocrine (33.17\%), and cardiac $(25.09 \%)$ disorders, and concomitant treatments to treat these diseases were reported consistently.

As expected, the group of patients with moderately severe $\mathrm{AD}$ had a significantly longer time of evolution (3.32 years versus 2.3 years in patients with mild or moderate $\mathrm{AD}$, $P<0.0001)$. However, no statistically significant association
Table I Sociodemographic and clinical characteristics of patients

\begin{tabular}{|c|c|}
\hline Characteristics & $\begin{array}{l}\text { Evaluable patients } \\
(n=1022)\end{array}$ \\
\hline Age (years), mean \pm SD & $78.4 \pm 6.62$ \\
\hline \multicolumn{2}{|l|}{ Gender, n (\%) } \\
\hline Male & $381(37.39)$ \\
\hline Female & $638(62.6 \mathrm{I})$ \\
\hline Weight (kg), mean \pm SD & $68.4 \pm 11.08$ \\
\hline Height $(\mathrm{cm})$, mean \pm SD & $163.5 \pm 8.82$ \\
\hline \multicolumn{2}{|l|}{ Blood pressure $(\mathrm{mmHg})$, mean $\pm \mathrm{SD}$} \\
\hline Systolic & $136.34 \pm 15.93$ \\
\hline Diastolic & $78.86 \pm 10.68$ \\
\hline \multicolumn{2}{|l|}{ Living situation, n (\%) } \\
\hline Alone & $95(9.42)$ \\
\hline Accompanied & $913(90.58)$ \\
\hline Availability of caregiver, $\mathrm{n}(\%)$ & $1010(99.02)$ \\
\hline Institutionalized patients, n (\%) & $175(17.17)$ \\
\hline Time of evolution of $A D$ (years), mean $\pm S D$ & $2.46 \pm 1.98$ \\
\hline Family history of $A D, n(\%)$ & $275(27.17)$ \\
\hline \multicolumn{2}{|l|}{ Severity of AD, n (\%) } \\
\hline Mild (MMSE score 24-2I) & $400(39.14)$ \\
\hline Moderate (MMSE score 20-15) & $457(44.72)$ \\
\hline Moderately severe (MMSE score 14-10) & $165(16.14)$ \\
\hline MMSE score, median (QI-Q3) & $20(16-22)$ \\
\hline Concomitant diseases, n (\%) & $838(82.00)$ \\
\hline Concomitant treatments, $\mathrm{n}(\%)$ & $801(78.38)$ \\
\hline \multicolumn{2}{|l|}{ Previous treatments for AD, n (\%) } \\
\hline Rivastigmine & $519(50.78)$ \\
\hline Capsules & $243(46.82)$ \\
\hline Oral solution & $272(52.4 I)$ \\
\hline Donepezil & $333(32.58)$ \\
\hline Donepezil ODT & $153(45.95)$ \\
\hline Standard tablets & $178(53.45)$ \\
\hline Galantamine & $147(14.38)$ \\
\hline Extended release capsules & $119(80.95)$ \\
\hline Standard tablets & $19(12.93)$ \\
\hline Oral solution & $7(4.76)$ \\
\hline Memantine & $49(4.79)$ \\
\hline \multicolumn{2}{|l|}{ Doses of ChEls (mg), mean \pm SD } \\
\hline Oral rivastigmine & $8.17 \pm 3.99$ \\
\hline Donepezil & $9.12 \pm 2.33$ \\
\hline Galantamine & $17.6 \pm 6.17$ \\
\hline
\end{tabular}

Abbreviations: AD, Alzheimer's disease; ChEls, cholinesterase inhibitors; MMSE, Mini-Mental State Examination; SD, standard deviation; QI, first quartile; Q3, third quartile; $\mathrm{ODT}$, orally disintegrating tablets.

was found between disease severity and family history of AD (Table 2). Among the complementary nonpharmacological interventions, cognitive stimulation $(24.27 \%)$, exercise $(18.88 \%)$, and memory training programs $(16.44 \%)$ were the most frequently prescribed.

The most frequent previous antidementia drug treatment was oral rivastigmine (taken by $50.78 \%$ of patients), followed by donepezil (32.58\%), and galantamine (14.38\%). Mean doses of cholinesterase inhibitors were $8.17 \mathrm{mg}$ (oral rivastigmine), $9.12 \mathrm{mg}$ (donepezil), and $17.6 \mathrm{mg}$ (galantamine); 
Table 2 Severity of AD depending on years of evolution and family history of the disease

\begin{tabular}{|c|c|c|c|c|}
\hline & \multirow[t]{2}{*}{ Statistic } & \multicolumn{3}{|c|}{ Severity of AD (MMSE score) } \\
\hline & & $\begin{array}{l}\text { Mild } \\
(24-2 I)\end{array}$ & $\begin{array}{l}\text { Moderate } \\
(20-15)\end{array}$ & $\begin{array}{l}\text { Moderately } \\
\text { severe }(14-10)\end{array}$ \\
\hline Time of AD evolution & $\mathrm{n}$ & 355 & 415 & 146 \\
\hline \multirow[t]{5}{*}{ (years) } & Mean (SD) & $2.3 \pm 2.03$ & $2.3 \pm 1.73$ & $3.32 \pm 2.27$ \\
\hline & Median & 2 & 2 & 3 \\
\hline & Minimum-maximum & $0-15$ & $0-11$ & $0-15$ \\
\hline & $\mathrm{Cl} 95 \%$ & $(2.09-2.51)$ & $(2.13-2.47)$ & $(2.95-3.69)$ \\
\hline & ANOVA ( $P$ value) & $<0.0001$ & & \\
\hline \multicolumn{5}{|l|}{ Family history of $A D$} \\
\hline Yes & $\mathrm{n}(\%)$ & $109(30.28)$ & $120(29.85)$ & $46(31.29)$ \\
\hline \multirow[t]{2}{*}{ No } & $\mathrm{n}(\%)$ & $25 I(69.72)$ & $282(70.15)$ & I0I (68.7I) \\
\hline & Chi-squared test ( $P$ value) & 0.9483 & & \\
\hline
\end{tabular}

Abbreviations: AD, Alzheimer's disease; ANOVA, analysis of variance; Cl, confidence interval; MMSE, Mini-Mental State Examination; SD, standard deviation.

$27.23 \%$ of patients were also treated with oral memantine (mainly tablets), at a mean dose of $19.65 \mathrm{mg}$ /day.

The switch to rivastigmine patches was mainly instigated on the initiative of the physician $(82.39 \%)$, and less frequently was due to a request or suggestion by the caregiver $(21.23 \%)$ or the patient $(10.37 \%$, Table 3$)$. A logistic regression analysis (Table 4) showed that, in comparison with previous treatment using donepezil, previous treatment with oral rivastigmine made the switch more likely to be instigated by the physician (odds ratio $1.68 ; 95 \%$ confidence interval $1.10-2.54, P=0.0145$ ). On the other hand, no significant difference was found between galantamine and oral rivastigmine with regard to the probability of switch on the initiative of the physician.

We observed that the increase in probability of switching prompted by patient request paralleled an increase in MMSE scores, so for each unit increase

Table 3 Promoters and reasons of switch to rivastigmine patches

\begin{tabular}{ll}
\hline & $\begin{array}{l}\text { Evaluable patients } \\
\text { (n = 1022) }\end{array}$ \\
\hline $\begin{array}{l}\text { Promoter of switch, } \mathrm{n}(\%) \\
\quad \text { Physician }\end{array}$ & $842(82.39)$ \\
$\quad$ Patient & $106(10.37)$ \\
$\quad$ Caregiver & $217(21.23)$ \\
Reason for switch, $\mathbf{n}(\%)$ & \\
Improving the ease of administration & $579(56.65)$ \\
Lack of efficacy/improving efficacy & $323(31.60)$ \\
Intolerability/improving tolerability & $376(36.79)$ \\
Poor adherence to treatment & $190(18.59)$ \\
(not due to intolerability) & \\
$\quad$ Physical diseases impairing administration & $19(1.86)$ \\
$\quad$ Rejection by the patient & $79(7.73)$ \\
$\quad$ Difficulty of medication management & $70(6.85)$ \\
Excessive caregiver burden & $58(5.67)$ \\
\hline
\end{tabular}

Note: Subjects were allowed to indicate several promoters and reasons. in MMSE score, a 16\% increase in the likelihood of a switch could be expected (odds ratio 1.16, $P=0.0001$ ). These results indicate that the lower the stage of disease severity, the higher the probability of a switch being made at the request of the patient. Similarly, we observed that the number of concomitant diseases increased the probability of switching due to patient request (odds ratio $1.43, P=0.0017)$. No statistically significant effects were observed when we analyzed the predictive factors of switch instigated by the caregiver.

Among the reasons for switching (Table 3), we found that improved convenience of administration was the most frequent reason, being reported by $56.65 \%$ of all patients, followed by poor tolerability (36.79\%), lack of efficacy or need to improve current treatment efficacy $(31.60 \%)$, and poor treatment adherence for reasons other than lack of tolerability (18.59\%). In the logistic regression analysis (Table 5), we found that previous treatment with oral rivastigmine increased the probability of switching to rivastigmine patches aimed at improving ease of administration when compared with previous treatment with donepezil or galantamine (odds ratio versus rivastigmine 0.24 for donepezil and 0.28 for galantamine; both $P<0.0001$ ). Likewise, previous treatment with standard galantamine tablets (two tablets daily) increased the probability of switching to rivastigmine patches in order to improve ease of administration, when compared with previous treatment using extended-release galantamine capsules (once a day). Conversely, no differences between orally disintegrating donepezil tablets and standard tablets were seen.

Quite the opposite was observed when the reason for the switch was lack of efficacy, where we found that previous treatment with donepezil or galantamine produced an approximately four-fold increase in the odds of switching 
Table 4 Results of the stepwise logistic regression analysis to find factors associated with different promoters for switch to rivastigmine patches

\begin{tabular}{|c|c|c|c|c|}
\hline & \multicolumn{3}{|l|}{ Odds ratio } & \multirow[t]{2}{*}{$P$ value } \\
\hline & Point estimate & Low $95 \% \mathrm{Cl}$ & Upper $95 \% \mathrm{Cl}$ & \\
\hline \multicolumn{5}{|l|}{ Physician as promoter of switch } \\
\hline \multicolumn{5}{|l|}{ Previous treatment for AD } \\
\hline Donepezil versus rivastigmine & 0.595 & 0.393 & 0.902 & 0.0145 \\
\hline Galantamine versus rivastigmine & 1.277 & 0.694 & 2.348 & 0.4318 \\
\hline Other versus rivastigmine & 1.824 & $0.4 I 5$ & 8.014 & 0.4262 \\
\hline \multicolumn{5}{|l|}{ Patient as promoter of switch } \\
\hline MMSE score & 1.163 & 1.078 & 1.255 & 0.0001 \\
\hline Number of concomitant diseases & 1.438 & 1.146 & 1.805 & 0.0017 \\
\hline Number of concomitant treatments & 0.661 & 0.528 & 0.828 & 0.0003 \\
\hline
\end{tabular}

Abbreviations: $\mathrm{AD}$, Alzheimer's disease; $\mathrm{Cl}$, confidence interval; MMSE, Mini-Mental State Examination.

compared with oral rivastigmine. No differences between orally disintegrating donepezil tablets and standard tablets nor between standard and extended-release galantamine were found for the probability of switching to transdermal rivastigmine.

No difference between previous treatments was found for the probability of switching to transdermal rivastigmine when the aim was to increase tolerability. Variables positively associated with switching due to treatment intolerability were increasing educational level of the caregiver and increasing number of concomitant diseases. Thus, the higher the caregiver's educational level, the higher the probability of switching for this reason, in particular, when the caregiver had completed secondary education (odds ratio for completed secondary studies versus no studies $1.67, P=0.0242$ ) and higher education (odds ratio for completed higher studies versus no studies 2.16, $P=0.0068$ ). Moreover, for an

Table 5 Results of the stepwise logistic regression analysis to find factors associated with different reasons for switch to rivastigmine patches

\begin{tabular}{|c|c|c|c|c|}
\hline & \multicolumn{3}{|l|}{ Odds ratio } & \multirow[t]{2}{*}{$P$ value } \\
\hline & Point estimate & Low $95 \% \mathrm{Cl}$ & Upper $95 \% \mathrm{Cl}$ & \\
\hline \multicolumn{5}{|l|}{ Reason for switch } \\
\hline \multicolumn{5}{|l|}{ Improving ease of administration } \\
\hline \multicolumn{5}{|l|}{ Previous treatment for $A D$} \\
\hline Donepezil versus rivastigmine & 0.238 & 0.167 & 0.337 & $<0.0001$ \\
\hline Galantamine versus rivastigmine & 0.281 & 0.183 & 0.432 & $<0.000$ I \\
\hline \multicolumn{5}{|l|}{ Reason for switch } \\
\hline \multicolumn{5}{|l|}{ Lack of efficacy } \\
\hline \multicolumn{5}{|l|}{ Previous treatment for $A D$} \\
\hline Donepezil versus rivastigmine & 4.303 & 2.957 & 6.261 & $<0.0001$ \\
\hline Galantamine versus rivastigmine & 3.928 & 2.505 & 6.160 & $<0.0001$ \\
\hline \multicolumn{5}{|l|}{ Reason for switch } \\
\hline \multicolumn{5}{|l|}{ Intolerability } \\
\hline \multicolumn{5}{|l|}{ Educational level of caregiver } \\
\hline Primary education versus no studies & $\mathrm{I} .340$ & $0.87 I$ & 2.060 & 0.1829 \\
\hline Secondary education versus no studies & 1.672 & 1.069 & 2.613 & 0.0242 \\
\hline Higher education versus no studies & 2.158 & 1.237 & 3.767 & 0.0068 \\
\hline Number of concomitant diseases & 1.116 & 1.001 & 1.246 & 0.0486 \\
\hline \multicolumn{5}{|l|}{ Reason for switch } \\
\hline \multicolumn{5}{|l|}{ Poor adherence } \\
\hline \multicolumn{5}{|l|}{ Educational level of patient } \\
\hline Primary education versus no studies & 0.551 & $0.36 \mathrm{I}$ & 0.840 & 0.0056 \\
\hline Secondary education versus no studies & 0.741 & 0.407 & 1.352 & 0.3286 \\
\hline Higher education versus no studies & 0.896 & 0.393 & 2.044 & 0.7948 \\
\hline Number of concomitant diseases & 1.250 & 1.098 & 1.424 & 0.0007 \\
\hline
\end{tabular}

Abbreviations: $\mathrm{AD}$, Alzheimer's disease; $\mathrm{Cl}$, confidence interval. 
increase in one unit in the number of concomitant diseases, an $11.6 \%$ increase in the odds of switching for this reason was expected.

Finally, when the reason for switching to transdermal rivastigmine was poor treatment adherence, no differences between previous treatments were found. Instead, we observed that the educational level of the patient, in particular having primary education, decreased the probability of switch due to poor treatment adherence (odds ratio for completed primary studies versus no studies $0.55, P=0.0056)$. Moreover, for an increase in one unit in the number of concomitant diseases, a $25 \%$ increase in the odds of presenting this reason was expected. In all cases, these models did not show lack of fit based on the Hosmer-Lemeshow test.

With regard to secondary variables, we found that $78.57 \%$ of patients started treatment with rivastigmine patches at a dose of $4.6 \mathrm{mg} /$ day and $95.08 \%$ were treated with $9.5 \mathrm{mg} /$ day during the continuation phase, with a median of 34 days from the starting dose to the highest dose, with a washout period in $17.22 \%$ of patients. In the logistic regression analysis, we found that the starting dose was influenced by the previous rivastigmine dose (with higher previous doses, there was a greater probability that the starting dose was $9.5 \mathrm{mg} /$ day, odds ratio $1.24, P<0.0001$ ) but no influence of previous doses of donepezil or galantamine was observed.

Finally, in the analysis to find relationships between establishing a washout period and the reasons for switching to rivastigmine patches, we found that establishing a washout period was related to switching to transdermal patches for intolerability reasons $(P<0.0001)$. Conversely, no statistically significant relationships were found between establishing a washout period and other reasons for switching, such as lack of efficacy or poor adherence.

\section{Discussion}

This study shows that most patients who switched to transdermal rivastigmine were previously receiving oral rivastigmine and that the change was mostly instigated by physicians, mainly in the search for improved ease of administration. In this regard, it seems feasible that physicians substituted oral rivastigmine for the transdermal formulation because conventional capsules or solution need to be taken twice daily with food, whereas the rivastigmine patch may be applied at any time of the day independently of meal times, making it a more convenient and flexible option. This assumption agrees with the fact that more changes to transdermal therapy from oral rivastigmine were seen compared with galantamine or donepezil when the reason for switching was ease of use, given that galantamine and donepezil are already available in convenient formulations that are easier to use than oral rivastigmine.

In contrast, switching to patches from galantamine or donepezil was four-fold more likely than switching from oral rivastigmine when the pursued benefit was increased efficacy. While the efficacy of rivastigmine is dose-dependent, transdermal delivery provides higher drug exposure but avoids the excessive peak plasma levels believed to cause adverse effects. Thus, patients not having clinical efficacy at low oral doses may benefit from switching to transdermal rivastigmine. Furthermore, a higher-dose rivastigmine patch $(13.5 \mathrm{mg} / 24$ hours, recently approved by the US Food and Drug Administration) was associated with significantly less deterioration in instrumental activities of daily living over a 48-week period, along with good tolerability, in deteriorating patients in comparison with nondecliners who were maintained on the $9.5 \mathrm{mg} / 24$-hour dose. ${ }^{11}$ Nevertheless, in our survey, physicians preferred to switch to another cholinesterase inhibitor when oral rivastigmine was found to be ineffective, rather than trying transdermal rivastigmine.

Unexpectedly, we did not find any statistically significant differences between any of the anticholinesterase drugs for the probability of switching to transdermal rivastigmine when intolerability or poor compliance was the reason for such a change. Some studies suggest that rivastigmine, when taken as oral capsules or solution, is not as well tolerated as donepezil or galantamine, with a higher incidence of gastrointestinal complaints. ${ }^{12,13}$ Nevertheless, transdermal rivastigmine was found to be noninferior to rivastigmine capsules, with a three-fold lower incidence of adverse gastrointestinal effects. ${ }^{3,14}$ Although the patches can produce skin irritation and allergic dermatitis, they seldom lead to treatment withdrawal. ${ }^{14}$ Besides, irritative dermatitis can be prevented by ensuring that the patch is applied to clean, dry skin and that it is not placed at the same application site within any 14-day period. Consequently, the tolerability of rivastigmine patches is suggested to be similar to that of donepezil or galantamine based upon manufacturers' product labeling data.

For all these reasons, we expected to find the main reason for switching from oral to transdermal rivastigmine to be poor tolerability. We also expected to see a higher probability of a switch to patches for this reason among patients on oral rivastigmine in comparison with those on galantamine or donepezil. Instead, we found that most switches from oral rivastigmine were instigated to achieve improved 
convenience rather than better tolerability and that previous medication did not predict switching implemented with the aim of increasing tolerability. One can then speculate that if oral rivastigmine is not tolerated, physicians prefer to switch to another class of anticholinesterase drug rather than to transdermal rivastigmine, and the same is possibly true for switches due to galantamine or donepezil intolerability.

With regard to switches aimed at overcoming noncompliance, this reason was only reported in $18.6 \%$ of cases. Again, no differences were seen between the anticholinesterase drugs. In this regard, the once-daily dosing scheme for the patch gives transdermal rivastigmine an advantage in terms of compliance over the twice-daily dosing schedule for oral rivastigmine. As has been previously reported, simplification of pharmacological regimens, together with educational interventions for patients and caregivers, a higher number of visits, and better communication between physicians and patients are usually associated with an improvement in treatment adherence. ${ }^{8,15}$ The transdermal delivery route provides some other advantages over oral forms drugs used to treat $\mathrm{AD}$ that could contribute to increased adherence and explain switching from galantamine or donepezil to improve compliance.

Other clinical variables influencing the switch were the patient's cognitive status and the presence of concomitant diseases. Thus, a relationship between better cognitive function and patient-instigated switching was reported. We also observed that the number of concomitant diseases was also related to the likelihood of switching prompted by patient request and due to intolerability and poor adherence, thus highlighting the inconveniences and potential dangers of polypharmacy reported for older patients ${ }^{16}$ and patients with dementia. ${ }^{17}$

Of note, we also identified some sociodemographic variables that played a role in determining the main reason for switching. Notably, a higher educational level of caregivers increased the probability of switching because of poor tolerability with previous medication. This finding, along with the fact that switching to patches was instigated by the caregiver in $21 \%$ of cases, shows the importance of caregiver education to assure optimal treatment. Likewise, a higher patient educational level was correlated with a lower switching rate due to poor treatment compliance. Finally, in the present study, we also found that the dosing pattern of transdermal rivastigmine used by physicians did not differ from the prescribing information.

\section{Conclusion}

Among the advantages the rivastigmine patch may offer, ease of use seems to be the one most clearly perceived by physicians. Accordingly, in everyday clinical practice, the main objective of neurologists when switching from oral to transdermal rivastigmine was convenience of use whereas when switching from galantamine or donepezil, the main purpose was to increase efficacy. No differences between previous treatments were seen in the probability of switching due to poor tolerability or treatment adherence. The educational level of patients and caregivers, as well as the number of concomitant diseases, were also involved in the decision to switch because of lack of tolerability or poor adherence.

\section{Acknowledgment}

The authors wish to thank Núria Piqué for her editorial assistance with this paper.

\section{Disclosure}

This study was funded by Esteve, SA, Barcelona, Spain. FJA is an employee of Esteve. SL-P has no conflict of interests to report in this work.

\section{References}

1. Popp J, Arlt S. Pharmacological treatment of dementia and mild cognitive impairment due to Alzheimer's disease. Curr Opin Psychiatry. 2011;24:556-561.

2. Farlow MR, Grossberg GT, Meng X, Olin J, Somogyi M. Rivastigmine transdermal patch and capsule in Alzheimer's disease: influence of disease stage on response to therapy. Int J Geriatr Psychiatry. 2011;26: 1236-1243.

3. Lee JH, Sevigny J. Effects of body weight on tolerability of rivastigmine transdermal patch: a post-hoc analysis of a double-blind trial in patients with Alzheimer disease. Alzheimer Dis Assoc Disord. 2011;25: 58-62.

4. Gauthier S, Juby A, Rehel B, Schecter R. EXACT: rivastigmine improves the high prevalence of attention deficits and mood and behaviour symptoms in Alzheimer's disease. Int J Clin Pract. 2007;61: 886-895.

5. Winblad B, Machado JC. Use of rivastigmine transdermal patch in the treatment of Alzheimer's disease. Expert Opin Drug Deliv. 2008;5: 1377-1386.

6. Cummings J, Winblad B. A rivastigmine patch for the treatment of Alzheimer's disease and Parkinson's disease dementia. Expert Rev Neurother. 2007;7:1457-1463.

7. Molinuevo JL, Arranz FJ. Impact of transdermal drug delivery on treatment adherence in patients with Alzheimer's disease. Expert Rev Neurother. 2012;12:31-37.

8. Molinuevo JL, Arranz FJ. Assessment of the strategies to improve therapeutic compliance in routine clinical practice in noncompliant patients with Alzheimer-type dementia. Rev Neurol. 2012;54:65-73.

9. Blesa R, Ballard C, Orgogozo JM, Lane R, Thomas SK. Caregiver preference for rivastigmine patches versus capsules for the treatment of Alzheimer disease. Neurology. 2007;69(4 Suppl 1):S23-S28.

10. Sevilla C, Jiménez-Caballero PE, Alfonso V. Orally disintegrating donepezil: are the main caregivers of patients with Alzheimer's disease more satisfied with this formulation of donepezil than with the traditional one? Rev Neurol. 2009;49:451-457.

11. Cummings J, Froelich L, Black SE, et al. Randomized, double-blind, parallel-group, 48-week study for efficacy and safety of a higher-dose rivastigmine patch (15 versus $10 \mathrm{~cm}^{2}$ ) in Alzheimer's disease. Dement Geriatr Cogn Disord. 2012;33:341-353. 
12. Bullock R, Touchon J, Bergman H, et al. Rivastigmine and donepezil treatment in moderate to moderately-severe Alzheimer's disease over a 2-year period. Curr Med Res Opin. 2005;21:1317-1327.

13. Lockhart IA, Mitchell SA, Kelly S. Safety and tolerability of donepezil, rivastigmine and galantamine for patients with Alzheimer's disease: systematic review of the 'real-world' evidence. Dement Geriatr Cogn Disord. 2009;28:389-403.

14. Winblad B, Cummings J, Andreasen N, et al. A six-month double-blind, randomized, placebo-controlled study of a transdermal patch in Alzheimer's disease - rivastigmine patch versus capsule. Int J Geriatr Psychiatry. 2007;22:456-467.
15. Guay DR. Rivastigmine transdermal patch: role in the management of Alzheimer's disease. Consult Pharm. 2008;23:598-609.

16. Monod S, Rochat S, Gold G, Büla C. Review in geriatric medicine. Rev Med Suisse. 2009;5:19-24. French.

17. Bassil N, Grossberg GT. Novel regimens and delivery systems in the pharmacological treatment of Alzheimer's disease. CNS Drugs. 2009; 23:293-307.

\section{Publish your work in this journal}

Patient Preference and Adherence is an international, peer-reviewed, open access journal focusing on the growing importance of patient preference and adherence throughout the therapeutic continuum. Patient satisfaction, acceptability, quality of life, compliance, persistence and their role in developing new therapeutic modalities and compounds to optimize clinical outcomes for existing disease states are major areas of interest. This journal has been accepted for indexing on PubMed Central. The manuscript management system is completely online and includes a very quick and fair peer-review system. Visit http://www.dovepress.com/ testimonials.php to read real quotes from published authors. 